\title{
Game Analysis on the Difficulties of Small and Medium-sized Enterprises' Credit Financing in the Big Data Era
}

\author{
Zhang Guowei ${ }^{1, a}$, Wang Tao ${ }^{2}$ \\ ${ }^{1}$ School of Economics, Harbin University of Commerce, Harbin, Heilongjiang, China \\ ${ }^{2}$ School of Economics, Harbin University of Commerce, Harbin, Heilongjiang, China
}

\begin{abstract}
The effective use of social funds is an important guarantee for the benign development of the economy. The shortage of funds faced by the development of small and medium-sized enterprises (SMEs) and a lot of social capital sits idle are still one of the major contradictions that need to be resolved urgently. And the main source of funds for China's real economy is still loans from commercial Banks. Through the analysis of the loan game between Banks and enterprises in the target economic environment we can find the crux of the problem. Which mainly lies in the market for enterprise information management demand and Market-based interest rate management to reduce market transaction costs and other regulatory construction issues. Therefore, in order to improve market transparency and reduce bank and corporate loan risks, the government and banks should make corresponding system management reforms in credit evaluation and classification and block management.
\end{abstract}

\section{INTRODUCTION}

The rise of emerging enterprises is one of the important ways to promote the efficient and rapid development of the social economy, which can stimulate the potential of society's potential for innovation, stabilize the social economy, and promote the adjustment of the economic structure. In order to comprehensively and scientifically evaluate the development and effectiveness of financial services for small and micro enterprises in commercial banks, to better play the role of regulatory incentives and guidance, and further improve the quality and efficiency of small and micro enterprises in commercial banks, in order to better solve these contradictions and problems, we need to make a systematic study of this to effectively promote the smooth progress of related work.

\section{ANALYSIS ON THE DIFFICULTIES OF SMALL BUSINESS LOANS}

Judging from the various efforts of the state and local governments, despite the introduction of a series of related policies, there are still difficulties in financing during the specific implementation process. Especially in the post-crisis period, on the one hand, there is a large amount of social idle funds, on the other hand, the contradiction of SMEs' lack of funds is widespread. The reasons for this are different, and there are mainly the following views:

\subsection{Principal agent}

Some scholars believe that excessive business risk of SMEs is the root cause of financing difficulties, which can be seen everywhere in a large number of literature on adverse selection and moral hazard. The SME guarantee system is not perfect, and credit and strength are difficult to meet the requirements of large credit (Tiyun Xia, 2017 ${ }^{[1]}$, The supply of funds is low, and China's financing guarantee business is unsustainable in the face of the new market situation (Wen Xuezhou 2019) ${ }^{[2]}$ Policy financing guarantees have become the backbone of the financing guarantee system, but the governance system is imperfect (Feng Beilin, 2020) ${ }^{[3]}$. But most of the analysis is not deep enough, and it is not close enough to our actual management.

\subsection{The lack of risk accounting}

Banks are unable to understand the development and credit status of SMEs in detail. Out of concerns about the repayment ability of enterprises (He Yan, 2013) ${ }^{[4]}$, even if the banks have sufficient funds, they are reluctant to lend money to venture companies. Credit risk control and management are related to the healthy growth of commercial banks and the stability of financial markets (Zhang Jinwei, 2013) $)^{[5]}$. This statement is closer to reality, but there is a lack of specific feasible solutions to improve this situation. 


\subsection{Information asymmetry}

Due to the asymmetric information between the bank and the enterprise, the company may violate the provisions of the loan contract signed with the bank, and the bank has the risk of losing principal and interest ( $\mathrm{Li}$ Wenjing, $2018)^{[6]}$.

\section{GAME ANALYSIS BASED ON MY COUNTRY'S BANK MANAGEMENT}

Loans from commercial banks are the main channel for corporate financing in my country. When many companies have a problem of poor capital turnover, they first thought of applying for loans from commercial banks. The main body involved in this business seems to be only the bank and the enterprise, that is, the bank will consider its risks, costs, time value and other issues when lending, while the enterprise mainly considers how to obtain loans, operating costs and operating risks. However, in this game, there are environmental issues such as government regulation and market success or failure, making our analysis not an isolated abstract game problem. To analyze this complex process clearly, you need to do the following specific analysis:

\subsection{Conflicts of interest indicated by simple static game between bank and enterprise}

Let the total capital required by the enterprise be $Y$, The expected annual rate of return is $r$ and the enterprise must have a certain "contingent" $\operatorname{cost} C_{e}$; the annual interest rate of the bank's loan is $i$, and the management fee after the bank loan is $C_{h}$; Before the loan is issued by the bank, the cost of the research work carried out by the enterprise is 0; Banks have two strategies to choose from-loans and loan rejections; Enterprises also have two strategies to choose from - keeping promises (repayment) and defaulting (non-repayment);

If the bank agrees to loan to the enterprise, and the enterprise also chooses to keep the contract and pay the loan in full and on time, then the bank's income is $Y i-C_{h}$ and the enterprise's income is $Y(1+r)-C_{e}$; If the bank refuses to loan, and the enterprise has made a plan to keep the contract, the revenue of both parties is $\left(0,-C_{e}\right)$; If the bank rejects the loan and the enterprise chooses not to repay, both gains are 0 . The static game process of the two parties is shown in the following table:

Table 1 Income payment matrix

\begin{tabular}{|c|c|c|c|}
\hline & & \multicolumn{2}{|c|}{ enterprise } \\
\hline & & keeping promises (repayment) & defaulting (non-repayment) \\
\hline \multirow[t]{2}{*}{ Bank } & Loan & $\left(Y i-C_{b}, Y(1+r)-C_{e}\right)$ & $\left(-Y-C_{b}, Y(1+r)-C_{e}\right)$ \\
\hline & rejects the loan & $\left(0,-C_{e}\right)$ & $(0,0)$ \\
\hline
\end{tabular}

In the actual game situation, due to the incompleteness and opacity of market information, or the deliberate concealment of one party, both parties do not fully understand the situation of the other party, especially the risk of banks is greater than that of enterprises. In the game, both parties believe that trustworthiness is a strategy with greater potential risks, and eventually uncooperative game results will appear. However, the game in reality is a dynamic process, which will have more complicated situations, and further analysis is needed.

\subsection{Dynamic game and its stability analysis}

In a purely free economy with no government, the strategy selection efficiency of enterprises and banks is low, which is not in line with the ideal state of both parties. Therefore, banks naturally need to add constraints in their management to protect their interests from being infringed, but the emergence of recourse rights makes the game appear in time sequence. Banks must first choose whether to lend, and the enterprise receives loans After deciding whether to keep the contract, in the event of a breach of the contract, the bank's recourse strategy is triggered, so that both parties will have some unnecessary losses. On this basis, under the assumptions of the static game, the following settings are made:

If the enterprise breaches the contract, the bank must initiate a recourse lawsuit with a cost of $K_{b}$; once the bank initiates a lawsuit, it will cause reputation loss $Q_{e}$ and collateral loss $P_{e}$; in the process of the game, the bank has no other costs except recourse costs;

$1-\omega$ is the probability of business failure during the loan period, $0<\omega<1 . \omega$ is the probability of business success; then the expected amount of money obtained by a bank is $\omega\left(Y i-C_{b}\right)+(1-\omega)\left(-Y-C_{b}-K_{b}\right)$, and the expected return of the enterprise is $\omega\left[Y(r-i)-C_{e}\right]+(1-\omega)\left[Y(1+r)-C_{e}-Q_{e}\right] \quad$, irrespective of who will bear the litigation cost of the bank;

Further suppose that the probability that the bank evaluates the profit of the enterprise (that is, the probability of being represented as a loan) is $\lambda$, then the probability of not lending to the enterprise will be $1-\lambda$; the probability of successful repayment of the business operation is $\omega$, and the probability of failure to repay the default business failure is $1-\omega$ the income payment matrix at this time is shown in the following table: 
Table 2 Income payment matrix

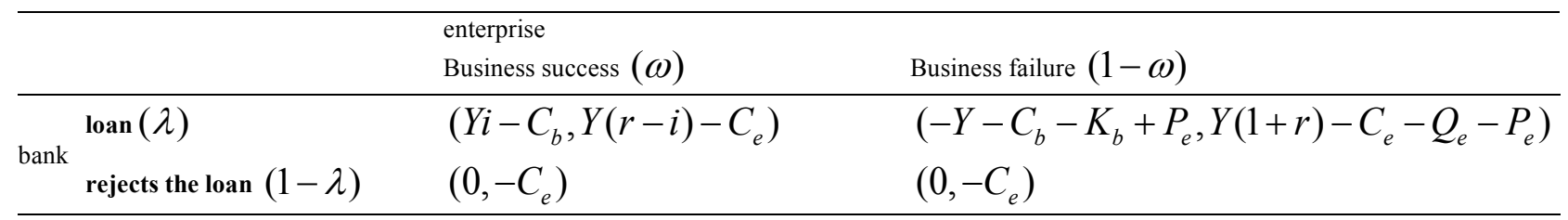

\subsubsection{Bank's Strategy Analysis and Stability}

The expected return of the bank on loan is:

$$
\begin{aligned}
& U_{1 b}=\omega\left(Y i-C_{b}\right)+(1-\omega)\left(-Y-C_{b}-K_{b}+P_{e}\right) \\
& =\omega Y(1+i)-\mathrm{Y}-C_{b}-(1-\omega) K_{b}+(1-\omega) P_{e}
\end{aligned}
$$

The expected return of the bank taking no loan is : $U_{2 b}=0$;

Then the average income of the bank is:

$U_{b}=\lambda U_{1 b}+(1-\lambda) U_{2 b}$

$$
\begin{aligned}
& =\lambda\left[\omega Y(1+i)-Y-C_{b}-(1-\omega) K_{b}+(1-\omega) P_{e}\right]+(1-\lambda) 0 \\
& =\lambda \omega Y(1+i)-\lambda Y-\lambda C_{b}-(1-\omega) \lambda\left(K_{b}+P_{e}\right)
\end{aligned}
$$

The bank's replication dynamic equation is

$$
\begin{aligned}
& \frac{d \lambda}{d t}=F(\lambda)=\lambda\left(U_{1 b}-U_{b}\right) \\
& =\lambda\left\{\left[\omega Y(1+i)-Y-C_{b}-(1-\omega) K_{b}+(1-\omega) P_{e}\right]\right. \\
& \left.-\left[\lambda \omega Y(1+i)-\lambda Y-\lambda C_{b}-(1-\omega) \lambda\left(K_{b}+P_{e}\right)\right]\right\} \\
& =\lambda(1-\lambda)\left[\omega Y(1+i)-Y-C_{b}-(1-\omega)\left(K_{b}-P_{e}\right)\right] \\
& =\lambda(1-\lambda)\left\{\omega\left[Y(1+i)+K_{b}-P_{e}\right]-Y-C_{b}-K_{b}+P_{e}\right\} \\
& \text { Let } \frac{d \lambda}{d t}=F(\lambda)=0 \text {, solve } \lambda_{1}=0, \quad \lambda_{2}=1,
\end{aligned}
$$

$\omega=\frac{Y+C_{b}+K_{b}-P_{e}}{Y(1+i)+K_{b}-P_{e}} \quad ;$ To analyze the copying dynamic equation of the bank's game subject, if $\omega^{*}=\frac{Y+C_{b}+K_{b}-P_{e}}{Y(1+i)+K_{b}-P_{e}}$, then $\frac{d \lambda}{d t}$ is always equal to 0 .This means that no matter what value $\lambda$ is taken at this time, it is a stable state, indicating that the greater the proportion of contingent $\operatorname{cost} C_{b}$ in interest $i$, the higher the probability of trust worthiness ; If $\omega^{*} \neq \frac{Y+C_{b}+K_{b}-P_{e}}{Y(1+i)+K_{b}-P_{e}}$, then $\lambda_{1}=0$ and $\lambda_{2}=1$ are two stable states, where

$$
\begin{aligned}
& \omega^{*}>\frac{Y+C_{b}+K_{b}-P_{e}}{Y(1+i)+K_{b}-P_{e}} \quad, \quad F(1)=0 \\
& F^{\prime}(1)<0 ; F(0)=0, \text { and } F^{\prime}(0)>0, \quad \lambda_{2}=1,
\end{aligned}
$$
so it is an evolutionarily stable strategy (ESS), indicating that the probability of success is greater than
$\frac{Y+C_{b}+K_{b}-P_{e}}{Y(1+i)+K_{b}-P_{e}}$, the bank must give loans ;

Similarly, where $\omega^{*}<\frac{Y+C_{b}+K_{b}-P_{e}}{Y(1+i)+K_{b}-P_{e}}, \lambda_{1}=0$ is

ESS, indicating that the probability of success is less than $\frac{Y+C_{b}+K_{b}-P_{e}}{Y(1+i)+K_{b}-P_{e}}$, the bank must not grant loans.

\subsection{2 enterprise's Strategy Analysis and Stability}

In the same way, the expected return on contract repayment after the successful operation of the enterprise is $U_{1 e}$, the expected return on failure to repay the enterprise is $U_{2 e}$, and the average return of the enterprise is $U_{e}$ :

$$
\begin{aligned}
& U_{1 e}=\lambda\left[Y(r-i)-C_{e}\right]+(1-\lambda)\left(-C_{e}\right)=\lambda Y(r-i)-C_{e} \\
& \quad U_{2 e}=\lambda\left[Y(1+r)-C_{e}-Q_{e}-P_{e}\right]+(1-\lambda)\left(-C_{e}\right) \\
& =\lambda Y(1+r)-C_{e}-\lambda Q_{e}-\lambda P_{e} \\
& U_{e}=\omega U_{1 e}+(1-\omega) U_{2 e} \\
& =\omega\left[\lambda Y(r-i)-C_{e}\right]+(1-\omega)\left[\lambda Y(1+r)-C_{e}-\lambda Q_{e}-\lambda P_{e}\right] \\
& =-\omega \lambda Y(1+i)+\lambda Y(1+r)-C_{e}-(1-\omega) \lambda\left(Q_{e}+P_{e}\right)
\end{aligned}
$$

Copy the dynamic differential equation as follows:

$$
\begin{aligned}
& \frac{d \omega}{d t}=G(\omega)=\omega\left(U_{1 e}-U_{e}\right) \\
& =\omega\left\{\left[\lambda Y(r-i)-C_{e}\right]-\right. \\
& \left.\left[-\omega \lambda Y(1+i)+\lambda Y(1+r)-C_{e}-(1-\omega) \lambda\left(Q_{e}+P_{e}\right)\right]\right\} \\
& =\omega(1-\omega)\left[\lambda Q_{e}-\lambda Y(1+i)-\lambda P_{e}\right]
\end{aligned}
$$

When $\frac{d \omega}{d t}=G(\omega)=0$, solve $\omega_{1}=0, \omega_{2}=1$, $\lambda^{*}=0$

When $\lambda^{*}=0, \frac{d \omega}{d t}==0$, all $\omega$ are in a stable state, that is, the bank does not make loans, and the enterprise cannot participate in the game, of course, it is impossible to test the integrity of the enterprise through the project; 
Assuming $Q_{e}-Y(1+i)-P_{e} \geq 0 \quad, \quad$ when $\lambda^{*} \neq 0, \quad G(1)=0, \quad G^{\prime}(1)<0 ; G(0)=0$ $G^{\prime}(0)>0$, so when $\lambda^{*} \neq 0, \omega_{2}=1$ is ESS. That is, when there are many $Q_{e}$, the company will keep its promise;

Assuming $Q_{e}-Y(1+i)-P_{e} \leq 0$, when $\lambda^{*} \neq 0, \quad G(0)=0, \quad G^{\prime}(0)<0 ; \quad G(1)=0$, $G^{\prime}(1)>0$, so when $\lambda^{*} \neq 0, \omega_{1}=0$ is ESS. That is, if $Q_{e}$ is small, the enterprise will not keep its promise.

\subsection{Dynamically evolved equilibrium analysis}

The dynamic evolution of the credit game between the bank and the enterprise is represented by a differential equation composed of $\frac{d \lambda}{d t}$ and $\frac{d \omega}{d t}$. According to the evolutionary stability, a stable state must be able to be stable to small disturbances, which can be used as the system's ESS .The dynamic equations of the system can be obtained from the replicator dynamic equations of the above bank and corporate financing

$$
\left\{\begin{array}{l}
F(\lambda)=\frac{d \lambda}{d t} \\
=\lambda(1-\lambda)\left\{\omega\left[Y(1+i)+K_{b}-P_{e}\right]-Y-C_{b}-K_{b}+P_{e}\right\} \\
G(\omega)=\frac{d \omega}{d t} \\
=\omega\left(U_{1 e}-U_{e}\right)=\omega(1-\omega)\left[\lambda Q_{e}-\lambda Y(1+i)-\lambda P_{e}\right]
\end{array}\right.
$$

When $\lambda$ and $\omega$ take different values, five local equilibrium points of the evolutionary game system can be obtained, $O(0,0), \quad A(1,0), \quad B(1,1), \quad C(0,1)$,
$D\left(\lambda^{*}, \omega^{*}\right)$. Where $\lambda^{*}=0$,

$\omega^{*}=\frac{Y+C_{b}+K_{b}-P_{e}}{Y(1+i)+K_{b}-P_{e}}$.

According to the stability of the equilibrium point of the differential equation evolution system, the local stability analysis of the Jacobian matrix of the game is calculated. $\frac{d \lambda}{d t}$ and $\frac{d \omega}{d t}$ differentiate $\lambda$ and $\omega$, respectively, to obtain the Jacobian matrix of the system game [4]:

$$
J=\left[\begin{array}{cc}
(1-2 \lambda)\left\{\omega\left[Y(1+i)+K_{b}-P_{e}\right]-Y-C_{b}-K_{b}+P_{e}\right\} & \lambda(1-\lambda)\left[Y(1+i)+K_{b}\right] \\
\omega(1-\omega)\left[Q_{e}-Y(1+i)\right] & (1-2 \omega)\left[\lambda Q_{e}-\lambda Y(1+i)-\lambda P_{e}\right]
\end{array}\right]
$$

Then the determinant and trace of the Jacobian matrix are:

$$
\operatorname{det} J=\left|\begin{array}{cc}
(1-2 \lambda)\left\{\omega\left[Y(1+i)+K_{b}-P_{e}\right]-Y-C_{b}-K_{b}+P_{e}\right\} & \lambda(1-\lambda)\left[Y(1+i)+K_{b}\right] \\
\omega(1-\omega)\left[Q_{e}-Y(1+i)\right] & (1-2 \omega)\left[\lambda Q_{e}-\lambda Y(1+i)-\lambda P_{e}\right.
\end{array}\right|
$$$$
\operatorname{tr} J=(1-2 \lambda)\left\{\omega\left[Y(1+i)+K_{b}-P_{e}\right]-Y-C_{b}-K_{b}+P_{e}\right\}+(1-2 \omega)\left[\lambda Q_{e}-\lambda Y(1+i)-\lambda P_{e}\right]
$$

Bring the five equilibrium points $\mathrm{O}, \mathrm{A}, \mathrm{B}, \mathrm{C}$, and $\mathrm{D}$ into the above matrix respectively to determine the local stability of each equilibrium point. Because of

$0 \leq \lambda^{*} \leq 1,0 \leq \omega^{*} \leq 1$, there is

$Y+C_{b} \leq Y(1+i)$,that is, $C_{b} \leq Y i$ 。 This is the final equilibrium of the bank, and the situation of the enterprise is divided into the following two situations:

\subsubsection{Equilibrium analysis when the reputation value is large}

The local stability of the system equilibrium point when $Q_{e}-Y(1+i)-P_{e} \geq 0$ is shown in the following table

Table 3 Stability analysis of equilibrium point

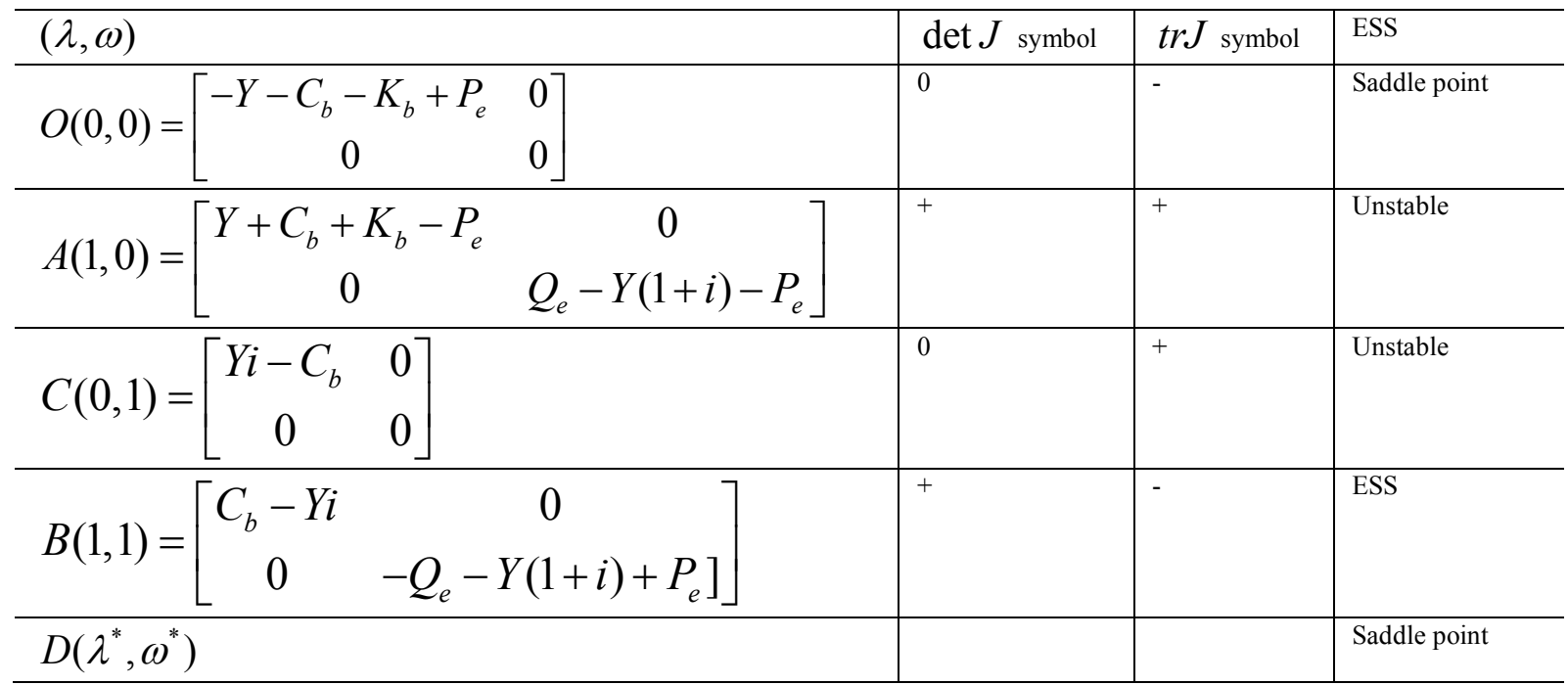


It can be seen from the above table that among the five equilibrium points, $B(1,1)$ has local stability (ESS), $Q_{e}-Y(1+i)-P_{e} \leq 0 \quad, \quad C(0,1) \quad$ are unstable equilibrium points, and the saddle point has $O(0,0), D\left(\lambda^{*}, \omega^{*}\right)$.From this, no matter what the value of $(\lambda, \omega)$ is, as long as $Q_{e}-Y(1+i)-P_{e} \geq 0$, it will tend to point $B(1,1)$ At this time, the bank loan and business operation are successfully repaid on time paragraph;

\subsubsection{Equilibrium analysis when the reputation value is small}

When the five equilibrium points of $Q_{e}-Y(1+i)-P_{e} \leq 0$ are $B(1,1), \quad A(1,0), C(0,1)$ are unstable equilibrium points, $O(0,0), \quad D\left(\lambda^{*}, \omega^{*}\right)$ is the saddle point, no point has ESS.

In this game, there is no ESS, but when $Q_{e}-Y(1+i)-P_{e} \leq 0$, it will tend to point $O(0,0)$, and the bank refuses the loan when making strategic decisions at this time;

It is concluded that the evolutionary stability strategy of this game is only point $B(1,1)$ when $Q_{e}-Y(1+i)-P_{e} \geq 0$, and all other points are not stable. Under the assumption of bounded rationality between the two sides of the game, according to the dynamic evolution of the credit of banks and enterprises, the following conclusions can be drawn: To achieve this strategy (bank loans, successful repayment of business operations on time), the prerequisite $Q_{e}-Y(1+i)-P_{e} \geq 0$ must be met.

\section{CONCLUSIONS}

In the above analysis, the key core elements are mainly $Y, i, Q_{e}, P_{e}$ and so on. When the loan limit $\mathrm{Yi}$ is smaller, $Q_{e}-Y(1+i)-P_{e}$ is larger; under the premise of marketization, the interest rate $i$ is the result of market equilibrium and is relatively fixed, but its size affects both banks and enterprises On the one hand, it is the income base of the bank, and on the other hand, the operating cost of the loan company.

The bank's credit rating to the enterprise is often consistent with the enterprise's credit, so for the enterprise, if the operation is successful, the greater the probability of repayment on time, the more the company's reputation loss $Q_{e}$ and collateral loss $P_{e}$ small. The regulatory treatment of these contradictions is the use of game mechanism design.

\subsection{The characteristic management reform of the banking system}

In order to improve the problem of difficulty and expensive financing for small businesses, the central bank proposed to implement targeted reduction of small and micro enterprises according to different banks, reduce the interest rate of technology-based small and micro enterprises loans, expand the loan quota of small and micro enterprises, and provide various guarantees for small and micro enterprises loans Principle preferential policies such as methods. However, in this context, can large commercial banks turn their attention to small and micro enterprises and actively respond to policies to support the development of small and micro enterprises? Need to do further analysis.

First, the investigation of corporate credit is one of the main costs of bank operation. Secondly, the probability of business success is determined. Third, the limited degree of marketization of interest rates has not served the purpose of regulating loan choices. Fourth, the coordinated use of other financial instruments. At present, there is almost no business insurance for small and medium-sized enterprises in the market. To reduce the bankruptcy crisis of small and medium-sized enterprises and increase trust in the operating capacity of enterprises, it is an important means to improve the financing ability of small and medium-sized enterprises.

\subsection{Government management reform}

In our game, banks and enterprises are relatively independent market players that seek to maximize profits, while government is the main body of market macro management. Some macro management carried out by the government aims to reduce the degree of information asymmetry in the market and the scale and scope of transaction costs. Therefore, the government should do the following two things:

First, accurately record all kinds of reputation information, and use the relevant social supervision mechanism to let the enterprise's goodwill affect its survival cost. Second, legal guarantees are used to reduce the uncertainty in various types of transactions, which also minimizes the transaction costs of the market and guarantees a virtuous cycle of the market.

\subsection{Management reform of the enterprise}

With the progress of economy and society, enterprises must not only be responsible for profits, but also be responsible for the environment and bear corresponding social responsibilities. The government must build social responsibilities of enterprises. While increasing profits, enterprises should pay more attention to environmental protection to achieve sustainable development and contribute to society. At the same time, for long-term development of an enterprise, it is necessary to enhance social responsibility, solve the problem of idle local labor, help underdeveloped areas to develop social undertakings, and also achieve advertising effects through public welfare undertakings, thereby gaining reputation and trust and achieving the company's longterm business goals. 


\section{REFERECES}

1. Ti Yunxia. Game Theory Analysis of Difficulty in Financing of Small and Medium-sized Enterprises and Difficulties in Bank Lending [J]. Learning from Accounting, 2017(13):215+217.

2. Wenzhou Zhou, Ding Xiaoli, Zhang Jing. Research on the Influencing Factors of the Financing Guarantee Effectiveness of Small and Micro Enterprises in China_Based on the Empirical Analysis of Jiangsu Guarantee Company [J]. Statistics and Information Forum, 2019, 34(01): 4957.

3. Feng Beilin. Research on precise measures to alleviate the difficulty of financing for enterprisesbased on surveys of financial institutions in Shanxi, Guangdong and Guizhou [J]. Economic Perspective, 2020(04):110-120.

4. He Yan. Research on the risk management model of microfinance companies [D]. Southwest Petroleum University, 2013.

5. Zhang Jinwei. Credit Game Analysis of Commercial Banks and Small and Micro Enterprises [J]. Special Zone Economy, 2013(09): 87-89.

6. Li Wenjing. The game between commercial banks and personal loan customers in the credit market [J]. Liaoning Economics, 2018(06): 25-27. 